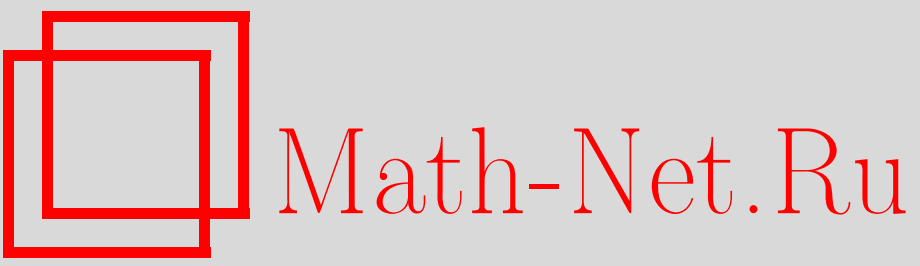

И. Л. Коган, Метод интеграла Дюамеля для обыкновенных дифференциальных уравнений с постоянными коэффициентами с точки зрения теории обобщенных функций, Вестн. Сам. гос. техн. ун-та. Сер. Физ.-мат. науки, 2010, выпуск 1(), 37-45

DOI: https://doi.org/10.14498/vsgtu673

Использование Общероссийского математического портала Math-Net.Ru подразумевает, что вы прочитали и согласны с пользовательским соглашением http: //www . mathnet.ru/rus/agreement

Параметры загрузки:

IP: 18.207 .199 .55

26 апреля 2023 г., 07:04:32 
УДК 517.926+517.982.45

\section{МЕТОД ИНТЕГРАЛА ДЮАМЕЛЯ ДЛЯ ОБЫКНОВЕННЫХ ДИФФЕРЕНЦИАЛЬНЫХ УРАВНЕНИЙ С ПОСТОЯННЫМИ КОЭФФИЦИЕНТАМИ С ТОЧКИ ЗРЕНИЯ ТЕОРИИ ОБОБЩЁННЫХ ФУНКЦИЙ}

\section{И. Л. Коган}

Российский государственный аграрный университет - МСХА им. К. А. Тимирязева, 127550, Москва, Тимирязевская ул., 49.

E-mail: ik_@list.ru

Приводится новое доказательство метода интеграла Дюамеля, основанное на свёрточной алгебре обобщённых функиий и позволяющее распространить этот метод на область отрицательных значений аргумента. Выведены универсальные формулы для решения уравнений с разрывной правой частью.

Ключевые слова: интеграл Дюамеля, пространство обобщённъх функиий, свёртка обобщённых функиий, алгебра свёртки.

1. Постановка задачи. Напомним суть метода, например [1, гл. VI]. Требуется найти решение линейного дифференциального уравнения с постоянными коэффициентами при нулевых начальных условиях

$$
L(D) y=f(x)
$$

где

$$
L(D)=D^{n}+a_{1} D^{n-1}+a_{2} D^{n-2}+\cdots+a_{n}=\sum_{i=0}^{n} a_{n-i} D^{i} \quad\left(a_{0}=1\right)
$$

- линейный дифференциальный оператор с постоянными коэффициентами, по известному решению $y_{1}(x)$ этого уравнения для специальной правой части $f(x)=1$, отвечающему также нулевым начальным условиям. Тогда искомое решение определяется сразу с помощью свёртки (интеграла Дюамеля)

$$
y(x)=\int_{0}^{x} f(\tau) y_{1}^{\prime}(x-\tau) d \tau
$$

или

$$
y(x)=y_{1}(x) f(0)+\int_{0}^{x} y_{1}(\tau) f^{\prime}(x-\tau) d \tau .
$$

С помощью интегрирования по частям из (3) следует (4) и наоборот. Воспользовавшись коммутативностью свёртки, можно получить ещё две эквивалентные формулы. Приведённое решение обычно выводится с помощью операционного исчисления, основанного на преобразовании Лапласа. Следовательно, исходя из требований, налагаемых на оригинал, $y(x)=0$ при $x<0$. В то же время решение, полученное для положительных значений $x$, возможно распространить и на всю ось $O x$.

Иосиф Леонидович Коган (к.т.н.), старший преподаватель, каф. высшей математики. 
Целью настоящей работы является обоснование метода интеграла Дюамеля непосредственно с помощью алгебры свёртки, в том числе, отдельно рассмотрев случай $x \leqslant 0$. Кроме того, удобно воспользоваться тем, что кусочно-гладкая функция, имеющая разрывы первого рода, с точки зрения теории обобщённых функций легко дифференцируется: в точках разрыва производная выражается через смещённую дельта-функцию Дирака. Последняя функция в свёрточной алгебре представляет оператор сдвига. Это позволяет получить универсальные формулы для решения уравнений с разрывной правой частью.

2. Новое доказательство формул (3) и (4). Пусть известно решение $y_{1}(x)$ дифференциального уравнения

$$
L(D) y_{1}(x)=1
$$

где оператор $L(D)$ определён формулой $(2)$, с нулевыми начальными условиями

$$
y_{1}(0)=y_{1}^{\prime}(0)=\cdots=y_{1}^{(n-1)}(0)=0 .
$$

Продифференцируем (5):

$$
\frac{d}{d x}\left(L(D) y_{1}(x)\right)=L(D) y_{1}^{\prime}(x)=0
$$

Отсюда следует, что $y_{1}^{\prime}(x)$ является решением однородного уравнения, причём, как видно из (6), отвечающим $n-1$ нулевым начальным условиям. Недостающее начальное условие для $\left(y_{1}^{\prime}\right)^{(n-1)}(0)=y_{1}^{(n)}(0)$ будет определено ниже.

Рассмотрим уравнение (5) в подпространстве обобщённых функций $D_{+}^{\prime}$ (с носителями на луче $x \in[0, \infty)$ ), образующих одноименную свёрточную алгебру [2, с. 141], [3, с. 87]:

$$
L(D) u_{1}(x)=\vartheta(x)
$$

где

$$
u_{1}(x)=\vartheta(x) y_{1}(x)
$$

и $\vartheta(x)$ - единичная функция Хевисайда.

Уравнение (7) в алгебре $D_{+}^{\prime}$ с единичным элементом $\delta(x)$ (дельта-функцией Дирака) имеет вид

$$
L\left(\delta^{\prime}\right) * u_{1}(x)=\vartheta(x)
$$

где $L\left(\delta^{\prime}\right)$ - свёрточный оператор, соответствующий $L(D)$.

Дифференцируем (8) по правилу дифференцирования обобщённых функций с учётом начальных условий (6):

$$
u_{1}^{(k)}(x)=\vartheta(x) y_{1}^{(k)}(x)(0 \leqslant k \leqslant n), \quad u_{1}^{(n+1)}(x)=\vartheta(x) y_{1}^{(n+1)}(x)+\delta(x) y_{1}^{(n)}(0) .
$$

Производные от $y_{1}(x)$ понимаются в обычном смысле. Теперь, используя (9), дифференцируем уравнение (7): 


$$
\begin{aligned}
& L(D) u_{1}^{\prime}(x)=L(D)\left(\vartheta(x) y_{1}^{\prime}(x)\right)+\delta(x) y_{1}^{(n)}(0)= \\
& \quad=\vartheta(x) L(D) y_{1}^{\prime}+\delta(x) y_{1}^{(n)}(0)=\delta(x) .
\end{aligned}
$$

Отсюда следует, что $u_{1}^{\prime}=\vartheta(x) y_{1}^{\prime}$ - фундаментальное решение. Так как $y_{1}^{\prime}(x)$, в свою очередь, является решением однородного уравнения, то для соблюдения равенства в (10) должно выполняться начальное условие $y_{1}^{(n)}(0)=1$. С учётом (6) функция $y_{1}^{\prime}(x)$ должна удовлетворять начальным условиям:

$$
y_{1}^{\prime}(0)=y_{1}^{\prime \prime}(0)=\cdots=y_{1}^{(n-1)}(0)=0, \quad y_{1}^{(n)}(0)=1 .
$$

Таким образом, $y_{1}^{\prime}(x)$ является решением однородного уравнения, отвечающим начальным условиям (11), и по теореме Лорана Шварца [2, с. 144] $\vartheta(x) y_{1}^{\prime}(x)$ представляет фундаментальное решение, а именно элементарное решение для оператора $L(D)$ или свёрточного оператора $L\left(\delta^{\prime}\right)$. Следовательно,

$$
\left(L\left(\delta^{\prime}\right)\right)^{-1}=\vartheta(x) y_{1}^{\prime}(x) .
$$

Неоднородное уравнение для оператора $L(D)$ с произвольной правой частью в алгебре $D_{+}^{\prime}$ имеет вид

$$
L\left(\delta^{\prime}\right) * y(x)=\vartheta(x) f(x),
$$

а его решение исходя из элементарного решения (12) для обычной локально интегрируемой функции $f(x)$ :

$$
y(x)=\left(L\left(\delta^{\prime}\right)\right)^{-1} * \vartheta(x) f(x)=\vartheta(x) y_{1}^{\prime}(x) * \vartheta(x) f(x)=\int_{0}^{x} f(\tau) y_{1}^{\prime}(x-\tau) d \tau .
$$

Таким образом, получена формула (3).

Найдём формулу (4) с помощью теории обобщённых функций (как отмечалось в п. 1, формулы (3) и (4) эквивалентны). Заметим, что элементарное решение (12), учитывая (9), можно представить как

$$
\left(L\left(\delta^{\prime}\right)\right)^{-1}=\left(\vartheta(x) y_{1}(x)\right)^{\prime} .
$$

Теперь воспользуемся полученным представлением (14) и правилом дифференцирования свёртки функций:

$$
y(x)=\left(\vartheta(x) y_{1}(x)\right)^{\prime} * \vartheta(x) f(x)=\vartheta(x) y_{1}(x) *(\vartheta(x) f(x))^{\prime},
$$

откуда получим формулу (4):

$$
y(x)=\vartheta(x) y_{1}(x) *\left(\vartheta(x) f^{\prime}(x)+f(0) \delta(x)\right)=y_{1}(x) f(0)+\int_{0}^{x} y_{1}(\tau) f^{\prime}(x-\tau) d \tau,
$$

так как $y_{1}(x)=\vartheta(x) y_{1}(x)$, если $x \geqslant 0$. При выводе формулы (4) получено полезное соотношение (15), выражающее решение в виде общего представления свёртки с производной от правой части уравнения (13). 
Решение неоднородного уравнения по формуле (3) или эквивалентным формулам (4), (15) имеет нулевые начальные условия (теорема Лорана Шварца). Впрочем, доказательство немедленно следует из (15). Дифференцируя эту формулу по правилу дифференцирования свёртки функций, имеем

$$
y^{(k)}(x)=\left(\vartheta(x) y_{1}(x)\right)^{(k)} *(\vartheta(x) f(x))^{\prime} .
$$

Из последней формулы, формул (6) и (9) вытекает это утверждение.

Теперь рассмотрим уравнение (5) в подпространстве $D_{-}^{\prime}$ (с носителями на луче $x \in(-\infty, 0])$, также образующих одноименную свёрточную алгебру [2, c. 146]:

$$
L(D) v_{1}(x)=\vartheta(-x), \quad \text { где } \quad v_{1}(x)=\vartheta(-x) y_{1}(x),
$$

или в алгебре $D_{-}^{\prime}$ :

$$
L\left(\delta^{\prime}\right) * v_{1}(x)=\vartheta(-x) .
$$

Проводя аналогичные выкладки, как и в случае $D_{+}{ }^{\prime}$, найдём элементарное решение для оператора $L(D)$ или свёрточного оператора $L\left(\delta^{\prime}\right)$ :

$$
\left(L\left(\delta^{\prime}\right)\right)^{-1}=-v_{1}^{\prime}(x)=-\vartheta(-x) y_{1}^{\prime}(x),
$$

где для $y_{1}^{\prime}(x)$ выполняются начальные условия $(11)$, т. е. $y_{1}^{(n)}(0)=1$. Неоднородное уравнение для оператора $L(D)$ с произвольной правой частью в алгебре $D_{-}^{\prime}$ имеет вид

$$
L\left(\delta^{\prime}\right) * y(x)=\vartheta(-x) f(x),
$$

а его решение исходя из выражения (16) для обычной локально интегрируемой функции $f(x)$ будет определяться формулой (3):

$$
\begin{aligned}
& y(x)=-\vartheta(-x) y_{1}^{\prime}(x) * \vartheta(-x) f(x)= \\
&=-\int_{x}^{0} f(\tau) y_{1}^{\prime}(x-\tau) d \tau=\int_{0}^{x} f(\tau) y_{1}^{\prime}(x-\tau) d \tau .
\end{aligned}
$$

Элементарное решение (16) можно выразить как

$$
\left(L\left(\delta^{\prime}\right)\right)^{-1}=-\left(\vartheta(-x) y_{1}(x)\right)^{\prime} .
$$

Учитывая (18), определим решение $y(x)$ для подпространства $D_{-}^{\prime}$ в виде, аналогичном (15):

$$
y(x)=-\left(\vartheta(-x) y_{1}(x)\right)^{\prime} * \vartheta(-x) f(x)=\vartheta(-x) y_{1}(x) *(-\vartheta(-x) f(x))^{\prime},
$$

откуда снова придём к формуле (4):

$$
\begin{aligned}
y(x)=\vartheta(-x) y_{1}(x) *(f(0) \delta(x)-\vartheta(-x) & \left.f^{\prime}(x)\right)= \\
& =y_{1}(x) f(0)+\int_{0}^{x} y_{1}(\tau) f^{\prime}(x-\tau) d \tau .
\end{aligned}
$$


Таким образом, формула (3) (или (4)) даёт решение линейного дифференциального уравнения с постоянными коэффициентами на всей числовой оси, удовлетворяющее нулевым начальным условиям.

3. Вопросы применения метода интеграла Дюамеля. Решение $y_{1}(x)$ вспомогательного уравнения (5) можно выразить через фундаментальное решение $\vartheta(x) u(x)$ для оператора $L(D)$ :

$$
y_{1}(x)=\vartheta(x) u(x) * \vartheta(x)=\int_{0}^{x} u(\tau) d \tau,
$$

где $u(x)$ - решение однородного уравнения $L(D) u=0$ с начальными условиями типа $(11): u^{(k)}(0)=0$ при $0 \leqslant k \leqslant n-2$ и $u^{(n-1)}(0)=1$.

Формулы (15) и (19), найденные для соответствующих подпространств, целесообразно применять при решении уравнения (1) с правой частью, имеющей разрывы первого рода, даже если для этого нужно предварительно определить $y_{1}(x)$. Преобразуем для этой цели указанные формулы.

Предложение 1. Решение уравнения (1) при $x \geqslant 0$ и нулевых начальных условиях, когда правая часть (13) $\vartheta(x) f(x)$ является кусочно-гладкой функиией с разрывами первого рода в точках $x_{i}$, определяется по формуле

$$
y(x)=\int_{0}^{x} y_{1}(\tau) f_{c l}^{\prime}(x-\tau) d \tau+\sum_{i} h_{i} \vartheta\left(x-x_{i}\right) y_{1}\left(x-x_{i}\right)
$$

или в развёрнутом виде

$$
\begin{aligned}
y(x)=\int_{0}^{x_{n}} y_{1}(\tau) f_{c l}^{\prime}(x-\tau) d \tau+\int_{x_{n}}^{x} y_{1}(\tau) f_{c l}^{\prime}(x- & \tau) d \tau+ \\
& +\sum_{i=0}^{n} h_{i} \vartheta\left(x-x_{i}\right) y_{1}\left(x-x_{i}\right),
\end{aligned}
$$

где $h_{i}=f\left(x_{i}+0\right)-f\left(x_{i}-0\right)-$ скачки в точках разрыва; $f_{c l}^{\prime}(x)-$ производная от $f(x)$ в классическом (обычном) смысле; $x_{0}=0, h_{0}=f(0) ; x_{n} \leqslant x<x_{n+1}$, $n \in \mathbb{N} \cup\{0\}$.

Действительно, дифференцируя $\vartheta(x) f(x)$ по правилу дифференцирования обобщённых функций, имеем

$$
\begin{aligned}
(\vartheta(x) f(x))^{\prime}=\vartheta(x) f_{c l}^{\prime}(x)+f(0) \delta(x)+ & \sum_{i(i \in \mathbb{N})} h_{i} \delta\left(x-x_{i}\right)= \\
& =\vartheta(x) f_{c l}^{\prime}(x)+\sum_{i(i=0)} h_{i} \delta\left(x-x_{i}\right) .
\end{aligned}
$$

Теперь, внося (22) в формулу (15) и используя свойство свёртки

$$
y(x) * \delta\left(x-x_{i}\right)=y\left(x-x_{i}\right),
$$

приходим к формуле (21). 
Предложение 2. Решение уравнения (1) при $x \leqslant 0$ и нулевых начальных условиях, если правая часть (17) $\vartheta(-x) f(x)$ является кусочно-гладкой функиией с разрывами первого рода в точках $x_{i}$, определяется по формуле

$$
y(x)=\int_{0}^{x} y_{1}(\tau) f_{c l}^{\prime}(x-\tau) d \tau+\sum_{i} h_{i}^{-} \vartheta\left(x_{i}-x\right) y_{1}\left(x-x_{i}\right)
$$

или

$$
\begin{aligned}
y(x)=\int_{0}^{x_{n}} y_{1}(\tau) f_{c l}^{\prime}(x-\tau) d \tau+\int_{x_{n}}^{x} y_{1}(\tau) f_{c l}^{\prime}(x-\tau) d \tau+ & \\
& +\sum_{i=0}^{n} h_{i}^{-} \vartheta\left(x_{i}-x\right) y_{1}\left(x-x_{i}\right),
\end{aligned}
$$

где $h_{i}^{-}=f\left(x_{i}-0\right)-f\left(x_{i}+0\right)-$ скачки в точках разрыва; $x_{0}=0, h_{0}=f(0)$; $x_{n} \geqslant x>x_{n+1}, n \in \mathbb{N} \cup\{0\}$.

Для обоснования (23) дадим вывод для выражения производной от $\vartheta(-x) f(x)$ в подпространстве $D_{-}^{\prime}$ несколько подробнее. Следуя аналогично $\left[4\right.$, c. 32], введём непрерывную функцию $f_{c l}(x)$, имеющую во всех точках гладкости $f(x)$ при $x \leqslant 0$ производную, совпадающую по значению с $f^{\prime}(x)$ :

$$
\vartheta(-x) f_{c l}(x)=\vartheta(-x) f(x)-\sum_{i(i \in \mathbb{N})} h_{i}^{-} \vartheta\left(x_{i}-x\right) .
$$

Дифференцируя это равенство и учитывая чётность дельта-функции, получаем

$$
\begin{aligned}
&(\vartheta(-x) f(x))^{\prime}=\vartheta(-x) f_{c l}^{\prime}(x)-f(0) \delta(x)-\sum_{i(i \in \mathbb{N})} h_{i}^{-} \delta\left(x_{i}-x\right)= \\
&=\vartheta(-x) f_{c l}^{\prime}(x)-\sum_{i(i=0)} h_{i}^{-} \delta\left(x_{i}-x\right) .
\end{aligned}
$$

Теперь, внося (24) в формулу (19) и используя $y(x) * \delta\left(x_{i}-x\right)=y(x) *$ $* \delta\left(x-x_{i}\right)=y\left(x-x_{i}\right)$, приходим к формуле $(23)$.

Далее приводим очевидное решение уравнения (1) с правой частью

$$
f(x)=\sum_{i} h_{i} \delta\left(x-x_{i}\right)
$$

где $h_{i}$ - постоянная и $x_{0}=0(i=0)$, найденное исходя из решения вспомогательного уравнения $y_{1}(x)$.

Предложение 3. Решение уравнения (1) при $x \geqslant 0$ и нулевых начальных условиях, если правая часть (13) представляет соотношение (25), находится по формуле

$$
y(x)=\sum_{i=0}^{n} h_{i} \vartheta\left(x-x_{i}\right) y_{1}^{\prime}\left(x-x_{i}\right),
$$


где $x_{n} \leqslant x<x_{n+1}$ u $n \in \mathbb{N} \cup\{0\}$.

Это выражение получено непосредственно из элементарного решения для оператора $L(D)$ в форме (12). Очевидно, что использование формулы (26) может быть целесообразным только в случае заданного выражения $y_{1}(x)$. В противном случае нужно найти элементарное решение исходя из решения однородного уравнения, как указано в начале этого пункта.

Пусть требуется определить решение исходного уравнения (1) при ненулевых начальных условиях в точке $x=0$. В этом случае к найденному решению с помощью метода интеграла Дюамеля, обладающему нулевыми начальными условиями, нужно прибавить решение однородного уравнения, отвечающего заданным начальным условиям.

Найдём решение (1) с начальными условиями

$$
y(0)=b_{0}, \quad y^{\prime}(0)=b_{1}, \quad \ldots, \quad y^{(n-1)}(0)=b_{n-1}
$$

по известному решению $y_{1}(x)$ вспомогательного уравнения $(5)$ с помощью теории обобщённых функций. Решение ищем в подпространстве $D_{+}^{\prime}$. Применяя оператор $L(D)$, заданный формулой $(2)$, к $\vartheta(x) y(x)$ и используя начальные условия (27) при вычислении производных от $y(x)$, приходим к уравнению [2, c. $144-145]$

$$
L(D)(\vartheta(x) y(x))=\vartheta(x) f(x)+\sum_{k=0}^{n-1} e_{k} \delta^{(k)}(x),
$$

где $e_{k}=b_{n-1-k}+a_{1} b_{n-2-k}+\cdots+a_{n-k-1} b_{0}$.

В алгебре $D_{+}^{\prime}$ уравнение $(28)$ имеет вид

$$
L\left(\delta^{\prime}\right) * \vartheta(x) y(x)=\vartheta(x) f(x)+\sum_{k=0}^{n-1} e_{k} \delta^{(k)}(x),
$$

откуда, используя представление для элементарного решения (12),

$$
y(x)=\vartheta(x) y_{1}^{\prime}(x) * \vartheta(x) f(x)+\sum_{k=0}^{n-1} e_{k} y_{1}^{(k+1)}(x) .
$$

Полученное решение (29) (по построению) удовлетворяет начальным условиям (27), свёртка в правой части (29) является одной из форм интеграла Дюамеля (15), т. е. представляет решение неоднородного уравнения (1) с нулевыми начальными условиями. Следовательно, слагаемое в виде суммы в этом выражении является решением однородного уравнения с заданными начальными условиями. С другой стороны, как показано в п. $\mathbf{2}, y_{1}^{\prime}(x)$ является решением однородного уравнения, это же относится и к производным более высокого порядка от $y_{1}(x)$ и линейным комбинациям этих производных. Таким образом, мы пришли к следующему предложению.

Предложение 4. Решение однородного уравнения $L(D) y_{0}(x)=0$, удовлетворяющее начальным условиям (27), определяется из решения $y_{1}(x)$ вспо- 
могательного уравнения (5) с нулевыми начальными условиями (6) по формуле

$$
y_{0}(x)=\sum_{k=0}^{n-1} e_{k} y_{1}^{(k+1)}(x),
$$

где е $e_{k}$ находятся согласно (28). Это решение, естественно, справедливо для любых значений $x$.

Примеры. Найти решение задачи Коши $y^{\prime \prime}+\omega^{2} y=f(x)$, если выполняются следующие условия:

1) $y(0)=y^{\prime}(0)=0$ и
a) $f(x)=\vartheta(x-a)-\vartheta(x-2 a)$;
б) $f(x)=\vartheta(x+a)-\vartheta(x-a)$;
в) $f(x)=\vartheta(x) \frac{x}{a}-\sum_{i=1}^{\infty} \vartheta(x-i a)(a>0)$;

2) $y(0)=b_{0}, y^{\prime}(0)=b_{1}$ и $f(x)$ - произвольная функция.

Решение. Частное решение исходного уравнения с $f(x)=1$ при нулевых начальных условиях легко найти из $(20)$ :

$$
y_{1}=\frac{1}{\omega^{2}}(1-\cos \omega x)
$$

1 а) Решение определяется по формуле (21). Здесь $h_{0}=f(0)=f_{c l}^{\prime}(x)=0$, $h_{1}=1$ при $x_{1}=a$ и $h_{2}=-1$ при $x_{2}=2 a$, поэтому

$$
y(x)=\vartheta(x-a) \frac{1}{\omega^{2}}(1-\cos \omega(x-a))-\vartheta(x-2 a) \frac{1}{\omega^{2}}(1-\cos \omega(x-2 a))
$$

или

$$
y(x)=\left\{\begin{array}{cc}
0, & x<a, \\
\frac{1}{\omega^{2}}[1-\cos \omega(x-a)], & a \leqslant x<2 a, \\
\frac{1}{\omega^{2}}[\cos \omega(x-2 a)-\cos \omega(x-a)], & x \geqslant 2 a .
\end{array}\right.
$$

1 б) Будем искать решения для подпространств $D_{+}^{\prime}$ и $D_{-}^{\prime}$ отдельно. В случае $D_{+}^{\prime}$ воспользуемся (21), и учитывая, что $h_{0}=f(0)=1, h_{1}=-1$ при $x_{1}=a, f_{c l}^{\prime}(x)=0$, получим

$$
y(x)=\vartheta(x) \frac{1}{\omega^{2}}(1-\cos \omega x)-\vartheta(x-a) \frac{1}{\omega^{2}}(1-\cos \omega(x-a))
$$

или

$$
y(x)=\left\{\begin{array}{cc}
\frac{1}{\omega^{2}}(1-\cos \omega x), & 0 \leqslant x<a, \\
\frac{1}{\omega^{2}}[\cos \omega(x-a)-\cos \omega x], & x \geqslant a .
\end{array}\right.
$$

В случае $D_{-}^{\prime}$ по (23), учитывая, что $h_{0}^{-}=f(0)=1, h_{1}^{-}=-1$ при $x_{1}=-a$, $f_{c l}^{\prime}(x)=0$, получим

$$
y(x)=\vartheta(-x) \frac{1}{\omega^{2}}(1-\cos \omega x)-\vartheta(-a-x) \frac{1}{\omega^{2}}(1-\cos \omega(x+a))
$$

или

$$
y(x)=\left\{\begin{array}{cc}
\frac{1}{\omega^{2}}(1-\cos \omega x), & -a<x \leqslant 0, \\
\frac{1}{\omega^{2}}[\cos \omega(x+a)-\cos \omega x], & x \leqslant-a .
\end{array}\right.
$$

Полученные решения для подпространств $D_{+}^{\prime}$ и $D_{-}^{\prime}$ можно свести в единую формулу

$$
y(x)=\left\{\begin{array}{cl}
\frac{1}{\omega^{2}}(1-\cos \omega x), & |x|<a, \\
\frac{1}{\omega^{2}}[\cos \omega(|x|-a)-\cos \omega x], & |x| \geqslant a .
\end{array}\right.
$$


1 в) Решение определяется по формуле (21). Здесь $h_{0}=f(0)=0, f_{c l}^{\prime}(x)=a^{-1}$ при $x \in[0, \infty), h_{i}=-1, x_{i}=i a, i \in \mathbb{N}$, поэтому

$$
y(x)=\frac{1}{\omega^{2}}\left[\frac{1}{a} \int_{0}^{x}(1-\cos \omega \tau) d \tau+\sum_{i=1}^{\infty} \vartheta(x-i a)(\cos \omega(x-i a)-1)\right] .
$$

Раскрывая это выражение, окончательно получим

$y(x)=\left\{\begin{array}{cc}\frac{1}{a \omega^{2}}\left(x-\frac{1}{\omega} \sin \omega x\right), & x<a, n=0, \\ \frac{1}{\omega^{2}}\left[\frac{1}{a}\left(x-\frac{1}{\omega} \sin \omega x\right)-n+\sum_{i=1}^{n} \cos \omega(x-i a)\right], n a \leqslant x<(n+1) a, n \in \mathbb{N} .\end{array}\right.$

2) Решение определяется по формуле (29), в которой для свёртки используется формула (15). Из формулы для $e_{k}$ в (28) при $n=2$ получаем $e_{0}=b_{1}$ $\left(a_{1}=0\right)$ и $e_{1}=b_{0}$, поэтому

$$
y(x)=\frac{\vartheta(x)}{\omega^{2}}(1-\cos \omega x) *(\vartheta(x) f(x))^{\prime}+b_{1} \frac{\sin \omega x}{\omega}+b_{0} \cos \omega x .
$$

БИБЛИОГРАФИЧЕСКИЙ СПИСОК

1. Лаврентъев М. А., Шабат Б. В. Методы теории функций комплексного переменного. М.: Наука, 1987. - 688 с.

2. Швари Л. Математические методы для физических наук. - М.: Мир, 1965. - 412 с.

3. Владимиров В. С. Обобщённые функции в математической физике. - М.: Наука, 1979. $320 \mathrm{c}$.

4. Гельфанд И. М., Шилов Г. Е. Обобщённые функции и действия над ними. Вып. 1. - М.: Добросвет, 2000. - 412 с.

Поступила в редакцию 20/VII/2009; в окончательном варианте - 12/II/2010.

MSC: 34L40, 47E05

\section{METHOD OF DUHAMEL INTEGRAL FOR ORDINARY DIFFERENTIAL EQUATIONS WITH CONSTANT COEFFICIENTS IN RESPECT TO THE THEORY OF DISTRIBUTIONS}

\section{L. Kogan}

Russian State Agrarian University - Moscow Agricultural Academy after K. A. Timiryazev, 49, Timiryazevskaya str., Moskow, 127550.

E-mail: ik_@list.ru

A new proof for the method of Duhamel integral is presented. This proof is based on the convolution algebra of distributions and allows to extend this method for the region $x<0$. Universal formulas for solving equations with discontinuous right-hand side are obtained.

Key words: Duhamel integral, space of distributions, convolution of distributions, convolution of distributions, convolution algebra.

Original article submitted 20/VII/2009; revision submitted $12 / \mathrm{II} / 2010$.

Iosif L. Kogan (Ph. D. (Techn.)), Lecturer, Dept. of Higher Mathematics. 